\title{
BMJ Open Levels of burn-out among healthcare workers during the COVID-19 pandemic and their associated factors: a cross-sectional study in a tertiary hospital of a highly burdened area of north-east Italy
}

\author{
Antonio Lasalvia (iD , , ${ }^{1,2}$ Francesco Amaddeo, ${ }^{2,3}$ Stefano Porru, ${ }^{4}$ Angela Carta, ${ }^{4}$
} Stefano Tardivo, ${ }^{5}$ Chiara Bovo, ${ }^{6}$ Mirella Ruggeri, ${ }^{1,2}$ Chiara Bonetto ${ }^{2}$

To cite: Lasalvia A, Amaddeo F, Porru S, et al. Levels of burn-out among healthcare workers during the COVID-19 pandemic and their associated factors: a cross-sectional study in a tertiary hospital of a highly burdened area of north-east Italy. BMJ Open 2021;11:e045127. doi:10.1136/ bmjopen-2020-045127

- Prepublication history and additional material for this paper are available online. To view these files, please visit the journal online (http://dx.doi. org/10.1136/bmjopen-2020045127).

Received 22 September 2020 Revised 31 December 2020 Accepted 06 January 2021

Check for updates

(c) Author(s) (or their employer(s)) 2021. Re-use permitted under CC BY-NC. No commercial re-use. See rights and permissions. Published by BMJ.

For numbered affiliations see end of article.

Correspondence to Dr Antonio Lasalvia; antonio.lasalvia@univr.it

\section{ABSTRACT}

Objective To determine burn-out levels and associated factors among healthcare personnel working in a tertiary hospital of a highly burdened area of north-east Italy during the COVID-19 pandemic.

Design Observational study conducted from 21 April to 6 May 2020 using a web-based questionnaire.

Setting Research conducted in the Verona University Hospital (Veneto, Italy).

Participants Out of 2195 eligible participants, 1961 healthcare workers with the full range of professional profiles (89.3\%) completed the survey.

Primary outcome measure Levels of burn-out, assessed by the Maslach Burnout Inventory-General Survey (MBI-GS). Multivariable logistic regression analysis was performed to identify factors associated with burn-out in each MBI-GS dimension (emotional exhaustion, EX; professional efficacy, EF; cynicism, $\mathrm{CY}$ ).

Results Overall, $38.3 \%$ displayed high EX, $46.5 \%$ low EF and $26.5 \%$ high CY. Burn-out was frequent among staff working in intensive care units (EX 57.0\%; EF 47.8\%; CY 40.1\%), and among residents (EX 34.9\%; EF 63.9\%; CY $33.4 \%$ ) and nurses (EX 49.2\%; EF 46.9\%; CY 29.7\%). Being a resident increased the risk of burn-out (by nearly 2.5 times) in all the three MBI subscales and being a nurse increased the risk of burn-out in the EX dimension in comparison to physicians. Healthcare staff directly engaged with patients with COVID-19 showed more EX and CY than those working in non-COVID wards. Finally, the risk of burnout was higher in staff showing pre-existing psychological problems, in those having experienced a COVID-related traumatic event and in those having experienced interpersonal avoidance in the workplace and personal life. Conclusions Burn-out represents a great concern for healthcare staff working in a large tertiary hospital during the COVID-19 pandemic and its impact is more burdensome for front-line junior physicians. This study underlines the need to carefully address psychological well-being of healthcare workers to prevent the increase of burn-out in the event of a new COVID-19 healthcare emergency.
Strengths and limitations of this study

- Strengths of this study include the large sample size, the representativeness of the sample with respect to the overall hospital population, the wide array of occupational profiles considered and data collection performed during the lockdown period of the pandemic.

- The relevance of this study should be also viewed in the light of the fact that it was conducted in the province of Verona (Veneto), one of the most burdened Italian areas, both in terms of deaths and infected COVID-19 cases.

- The response rate was relatively low (however, literature reports that web-based surveys conducted among physicians and healthcare workers have generally response rates similar to that of our study).

- Personality traits or individual psychological characteristics were not considered among possible predictors.

- Other contextual and organisational variables that might have exerted a significant role in the prediction of burn-out were not included in the analyses.

- The cross-sectional nature of this survey did not allow for the determination of causal relationships.

\section{INTRODUCTION}

Since the beginning of the COVID-19 pandemic, healthcare workers have been under heavy workload conditions worldwide. Limited resources, longer shifts, disruptions to sleep and work-life balance, and occupational hazards associated with exposure to patients with COVID-19 have contributed to adverse psychological outcomes among healthcare workers in terms of post-traumatic stress, insomnia, anxiety and depression. ${ }^{1-3}$ The COVID-19 pandemic may have also negatively impacted healthcare workers in 
terms of increased burn-out. ${ }^{4}$ Burn-out is a psychological syndrome developing as a negative reaction to occupational stressors, ${ }^{5}$ composed by a combination of emotional exhaustion, depersonalisation and low personal accomplishment. ${ }^{6}$ Emotional exhaustion is related to an individual's experience of stress, which is, in turn, related to a decline in emotional and physical resources. Depersonalisation (or cynicism) refers to detachment from work in reaction to the overload of exhaustion and pertains to the loss of enthusiasm and passion for one's work. ${ }^{7}$ Personal accomplishment refers to the feelings of low professional efficacy and lack of productivity at work. ${ }^{7}$ The consequences of burn-out are not restricted to the workers' health, but also affect the quality of care provided and the organisational well-being. ${ }^{8}$

Before the pandemic, the clinical workforce in most Western countries had experienced a rise in burn-out. ${ }^{4}$ The COVID-19 pandemic may have exacerbated an already existing problem within overstretched healthcare systems, thus further increasing the pernicious effects of burn-out. During the COVID-19 pandemic burn-out has been less extensively explored than other psychological outcomes. The limited available literature is still conflicting. A survey conducted in China found a high prevalence of burn-out among front-line nurses. ${ }^{9}$ Conversely, another study also conducted in China found that physicians and nurses working in COVID-19 wards had lower frequencies of burn-out than those working in usual wards. ${ }^{10}$ Another study conducted on Italian frontline healthcare workers found that the percentage of those reporting high levels of burn-out was higher than the one found in other Italian samples before the pandemic ${ }^{11}$; however, the generalisability of findings is questionable, since the study was conducted on a convenience sample of people using social media. Finally, a study carried out in the University Hospital of Augsburg (Germany) found that nurses working in COVID-19 wards had higher levels of burn-out compared with their colleagues working in usual wards, whereas no difference in terms of burn-out was found between physicians working in COVID-19 wards with respect to those of usual wards ${ }^{12}$; the generalisability of these findings, however, was also limited due to a relatively small sample size and the fact that no information was given on the representativeness of the sample with respect to the overall hospital staff.

The present study was undertaken aiming to: (1) assess the extent of burn-out in a large representative sample of healthcare personnel working in the Verona University Hospital during the COVID-19 pandemic, and (2) explore factors associated to burn-out by considering both personal and work-related characteristics, together with COVID-related factors.

\section{METHODS}

\section{Study design}

This study was conducted within the context of an ongoing longitudinal project aiming to assess the psychological impact of COVID-19 among Verona University Hospital workers during the lockdown phase, after 2 months and at 1 year. ${ }^{13}$ Data collection was performed from 21 April to 6 May 2020 using a web-based questionnaire hosted on the online platform 'SurveyMonkey'. The study description, the invitation to participate and the link to the online questionnaire were published in the hospital's newsletter and sent via email by the trust administration to all hospital workers. The survey was anonymous, and confidentiality of information was ensured.

\section{Setting and participants}

The Verona University Hospital is a large academic hospital located in Veneto, the first Italian region (with Lombardy) hit by the pandemic and one of the most burdened Italian regions in terms of infected persons and deaths for COVID-19. It is the second largest hospital trust in Italy in terms of beds and the fifth largest in terms of admissions. The hospital staff is composed of 5940 personnel (including nearly 1200 residents of the medical specialty schools at the University of Verona). All health and administrative employees working in the Verona University Hospital during the lockdown phase of the pandemic were asked to participate in the study.

\section{Outcomes and covariates}

Burn-out was assessed by the Maslach Burnout InventoryGeneral Survey (MBI-GS). ${ }^{14}$ The MBI-GS is a modified version of the original $\mathrm{MBI}^{6}$ that was specifically designed to assess burn-out in any occupational setting. We decided to use the MBI-GS, as this scale is based on the assumption that burn-out takes the same form in all occupational sectors and it is related to the general performance of work, rather than on relationships at work (eg, with patients) - that is, burn-out can occur anytime and anywhere there is a major imbalance, or mismatch, between demands in the work environment and the individual's available resources. ${ }^{7}$ The MBI-GS was found to be reliable and valid across multiple cultural settings and occupations, including healthcare professionals. ${ }^{15-18}$ It consists of 16 items constituting three subscales that parallel those of the original MBI: emotional exhaustion (EX; 5 items) covers the experience of both emotional and physical fatigue; cynicism (CY; 5 items) reflects indifference, detached attitude towards work and active disengagement from work; professional efficacy (EF; 6 items) consists of feelings of competence, successful achievement and accomplishment in one's work, which diminish when burn-out is developing. All MBI-GS items are scored on a 7-point rating scale ranging from 0 (never) to 6 (always). Since the MBI-GS authors ${ }^{19}$ recommend not to use cut-off scores obtained in one country to classify subjects in another county, we used the only Italian cut-off scores available in the literature for the healthcare sector ( $>2.20$ for EX, $>2.00$ for $\mathrm{CY}$ and $<3.66$ for EF); these cutoff scores were tested on a large sample of mental health professionals working in the Veneto region. ${ }^{20}$ 
Personal background information and job-related factors were collected, including sex, age, living condition, occupation, having pre-existing psychological problems (ie, developed before the COVID-19 outbreak) requiring specialised help, length of working experience and place of work (hospital unit). For the purpose of analysis, the various hospital units were stratified according to the degree of clinical engagement with patients with COVID-19, from most engaged to least engaged: intensive care units (ICU; that during the lockdown phase were entirely dedicated to critically ill patients with COVID19), subintensive COVID wards (ie, infectious disease, pulmonary medicine and internal medicine wards specifically dedicated to COVID-19), front-line services dealing with patients with COVID-19 (ie, radiology and emergency department), non-COVID wards, laboratory diagnostic services (ie, laboratory medicine, transfusion medicine, immunology, pathology, microbiology) and administration.

COVID-19 work-related information was also collected. Specifically, participants were questioned on the occurrence of related traumatic experiences at work, their personal condition ('in isolation as tested positive or hospitalisation for COVID-19' vs none), if they had to move out of the family house to protect family members and if they had to make changes to their personal habits or lifestyle. Finally, three ad hoc schedules were administered exploring: (1) COVID-19-related job stress ${ }^{21}$ ('There was more conflict among colleagues at work'; 'I had to do work that normally I do not do'; 'I had an increased workload'); (2) COVID-19-related perception of risk in the workplace ('I shouldn't be looking after patients with COVID-19' (avoidance of patient); 'I accept the risk of getting COVID-19 as part of my job' (acceptance of risk); 'I am afraid of falling ill with COVID-19' (fear)); (3) COVID-19-related perceived interpersonal avoidance ${ }^{22}$ ('I thought that people avoided me because of my profession', 'I thought that people avoided my family because of my profession', 'I thought that my family avoided me because of my profession') and avoidant safety behaviour ('I avoided colleagues who might be exposed to COVID19 '; 'I was afraid to infect my family members when going back home from work'). For the purpose of analysis, the three items exploring perceived interpersonal avoidance were collapsed into one 'perceived avoidance scale' and the two items of avoidant safety behaviours into a 'safety behaviour scale', both ranging from ' 0 ' (no perceived interpersonal avoidance/no avoidant safety behaviours) to '4' (high perceived interpersonal avoidance/high avoidant safety behaviours).

\section{Statistical analysis}

Statistical analyses were carried out using SPSS V.22 and Stata V.15. Descriptive statistics were given by frequencies and percentages. Comparisons between categorical variables were performed by $\chi^{2}$ or Fisher's exact tests, where appropriate. The association between each burn-out domain (emotional exhaustion, cynicism and professional efficacy) and each potential risk factor selected a priori, on clinical or empirical grounds and derived from the relevant literature, was explored by estimating unadjusted ORs and 95\% CIs using univariate logistic regression models. Subsequently, multivariate logistic regression models for the same outcomes gave adjusted ORs and 95\% CIs. Goodness-of-fit measures were estimated for these models. The alpha level was set to 0.05 for all effects.

\section{Patient and public involvement}

Patients and/or the public were involved in the design, or conduct, or reporting, or dissemination plans of this research.

\section{RESULTS}

\section{Personal and work-related characteristics}

Overall, 2195 workers (36.9\% of the eligible population) participated in the online survey. The representativeness of participants was assessed by comparing the study sample with the overall eligible population on two key characteristics for the aims of this study and for which official statistics from the Verona University Hospital were available, that is, occupation and exposure to patients with COVID-19. For details, see online supplemental part 1. Overall, the study sample overlapped with the Verona University Hospital staff both in terms of occupational profile composition and percentage of healthcare workers employed in units directly engaged with patients with COVID-19, thus indicating that selection of participants did not produce a biased sample. The sample addressed here may be therefore considered representative of the overall hospital population.

MBI-GS was completed by 1961 out of 2195 (89.3\%) healthcare workers. Completers differed from noncompleters $(n=234)$ only in terms of occupational profile and exposure to COVID-related traumatic events. Specifically, physicians $(93.5 \%$ completers vs $6.5 \%$ noncompleters) and residents (92.8\% completers vs $7.2 \%$ non-completers) were more likely to complete the MBI-GS compared with nurses $(87.7 \%$ completers vs $12.3 \%$ non-completers), other healthcare staff $(84.7 \%$ completers vs $12.6 \%$ non-completers) and administrative staff (88.2\% completers vs $11.8 \%$ non-completers) $\left(\chi^{2}\right.$ test, $\mathrm{p}=0.003)$. Moreover, staff not exposed to COVIDrelated traumatic events were more likely to complete the MBI-GS (96.0\% completers vs $4.0 \%$ non-completers) compared with exposed staff ( $88.3 \%$ completers vs $11.7 \%$ non-completers) (Fisher's exact test, $\mathrm{p}<0.001$ ).

Personal and work-related characteristics, together with COVID-19 work-related information within the study sample, are detailed in table 1.

\section{Job stress, perception of risk and interpersonal avoidance among healthcare workers}

As shown in table 2, half of the sample perceived more conflicts among colleagues, $59.6 \%$ reported that they 
Table 1 Personal and job characteristics of participants who completed the MBI-GS $(n=1961)$

\begin{tabular}{|c|c|c|}
\hline & $\mathrm{n}$ & $\%$ \\
\hline \multicolumn{3}{|l|}{ Sex (6 missing) } \\
\hline Male & 492 & 25.2 \\
\hline Female & 1463 & 74.8 \\
\hline \multicolumn{3}{|l|}{ Age (5 missing) } \\
\hline$<36$ years & 633 & 32.4 \\
\hline $36-55$ years & 980 & 50.1 \\
\hline$>55$ years & 343 & 17.5 \\
\hline \multicolumn{3}{|l|}{ Living condition (6 missing) } \\
\hline Alone & 322 & 16.5 \\
\hline With family/other relatives & 1633 & 83.5 \\
\hline \multicolumn{3}{|c|}{ Length of working experience (13 missing) } \\
\hline$<6$ years & 594 & 30.5 \\
\hline 6-20years & 593 & 30.4 \\
\hline >20years & 761 & 39.1 \\
\hline \multicolumn{3}{|l|}{ Occupation } \\
\hline Physicians & 286 & 14.6 \\
\hline Residents & 335 & 17.1 \\
\hline Nurses & 687 & 35.0 \\
\hline Other healthcare staff & 466 & 23.8 \\
\hline Administrative staff & 187 & 9.5 \\
\hline \multicolumn{3}{|l|}{ Workplace (31 missing) } \\
\hline Intensive care units & 182 & 9.4 \\
\hline $\begin{array}{l}\text { Subintensive care wards for } \\
\text { patients with COVID-19* }\end{array}$ & 164 & 8.5 \\
\hline $\begin{array}{l}\text { Front-line services dealing with } \\
\text { patients with COVID-19† }\end{array}$ & 146 & 7.6 \\
\hline Non-COVID wards & 1062 & 55.0 \\
\hline Laboratory diagnostic servicesł & 216 & 11.2 \\
\hline Administration & 160 & 8.3 \\
\hline
\end{tabular}

Having pre-existing psychological problems

\begin{tabular}{|c|c|c|}
\hline Yes & 120 & 6.1 \\
\hline No & 1841 & 93.9 \\
\hline \multicolumn{3}{|c|}{ Experienced COVID-related traumatic event } \\
\hline No & 759 & 38.7 \\
\hline Yes & 1202 & 61.3 \\
\hline \multicolumn{3}{|c|}{ Personal condition in relation to COVID-19 } \\
\hline $\begin{array}{l}\text { Isolation as tested positive/ } \\
\text { hospitalisation for COVID }\end{array}$ & 295 & 15.0 \\
\hline None & 1666 & 85.0 \\
\hline \multicolumn{3}{|c|}{$\begin{array}{l}\text { Move out of the family house to protect family members ( } 12 \\
\text { missing) }\end{array}$} \\
\hline No & 1762 & 90.4 \\
\hline Yes & 187 & 9.6 \\
\hline \multicolumn{3}{|c|}{ Changes in personal lifestyle due to COVID-19 (18 missing) } \\
\hline No & 885 & 45.5 \\
\hline
\end{tabular}

Continued
Table 1 Continued

\begin{tabular}{llc}
\hline & $\mathrm{n}$ & $\%$ \\
\hline Yes & 1058 & 54.5 \\
\hline
\end{tabular}

*Infectious disease unit, pulmonary medicine, internal medicine units converted specifically to COVID-19.

†Radiology and emergency department.

‡Laboratory medicine, transfusion medicine, immunology, pathology, microbiology.

MBI-GS, Maslach Burnout Inventory-General Survey.

had to do work they usually were not supposed to do and $65.3 \%$ had an increased workload.

As regards the perception of risk, $66.5 \%$ reported having felt uncomfortable when looking after patients with COVID-19 and $82.8 \%$ were afraid to get infected by SARS-CoV-2, even though $90 \%$ accepted the risk of getting COVID-19 as part of their work. Regarding interpersonal avoidance, $49.6 \%$ of hospital staff reported having been avoided by other people because of their profession, $26.7 \%$ reported their family members had been avoided for the same reason, $28.5 \%$ avoided at work colleagues who might have been exposed to patients with COVID-19 and $64.9 \%$ were afraid to infect their family members once back at home.

\section{Prevalence of burn-out among healthcare workers}

Overall, $38.3 \% \quad(\mathrm{n}=752)$ of participants displayed symptoms of high emotional exhaustion (EX), 46.5\% ( $\mathrm{n}=911)$ of low professional efficacy (EF) and 26.5\% ( $\mathrm{n}=519)$ of high cynicism (CY).

As shown in table 3, prevalence of burn-out was high among staff working in both ICUs (EX 57.0\%; EF 47.8\%; CY $40.1 \%$ ) and subintensive care wards for patients with COVID-19 (EX 53.0\%; EF 47.6\%; CY 22.6\%) and, with respect to the occupational profile, among residents (EX 34.9\%; EF 63.9\%; CY 33.4\%) and nurses (EX 49.2\%; EF $46.9 \%$; CY 29.7\%).

Burn-out was also more frequent among healthcare workers who had been in treatment for psychological problems (EX 58.3\%; EF 57.5\%; CY 39.2\%) and among those who had experienced a COVID-related traumatic event at work (EX 49.8\%; EF 48.3\%; CY 30.3\%). Finally, burn-out was frequent among healthcare workers who had decided to move out of the family house to protect other family members from a possible contagion (EX $46.5 \%$; EF $54.5 \%$; CY 34.2\%) and those who had to change their personal habits and lifestyle due to COVID-19 (EX $43.7 \%$ ).

Details on job stress, perception of risk and interpersonal avoidance across the three burn-out domains are given in the online supplemental part 2. In brief, burn-out (particularly in its EX component) was more frequent among healthcare workers who had reported higher stress at work, those who perceived themselves to be at higher risk of contagion and those who had experienced social avoidance because of their profession. 
Table 2 Job stress, perception of risk and interpersonal avoidance in the participants assessed by MBI-GS (n=1961)

$\%$

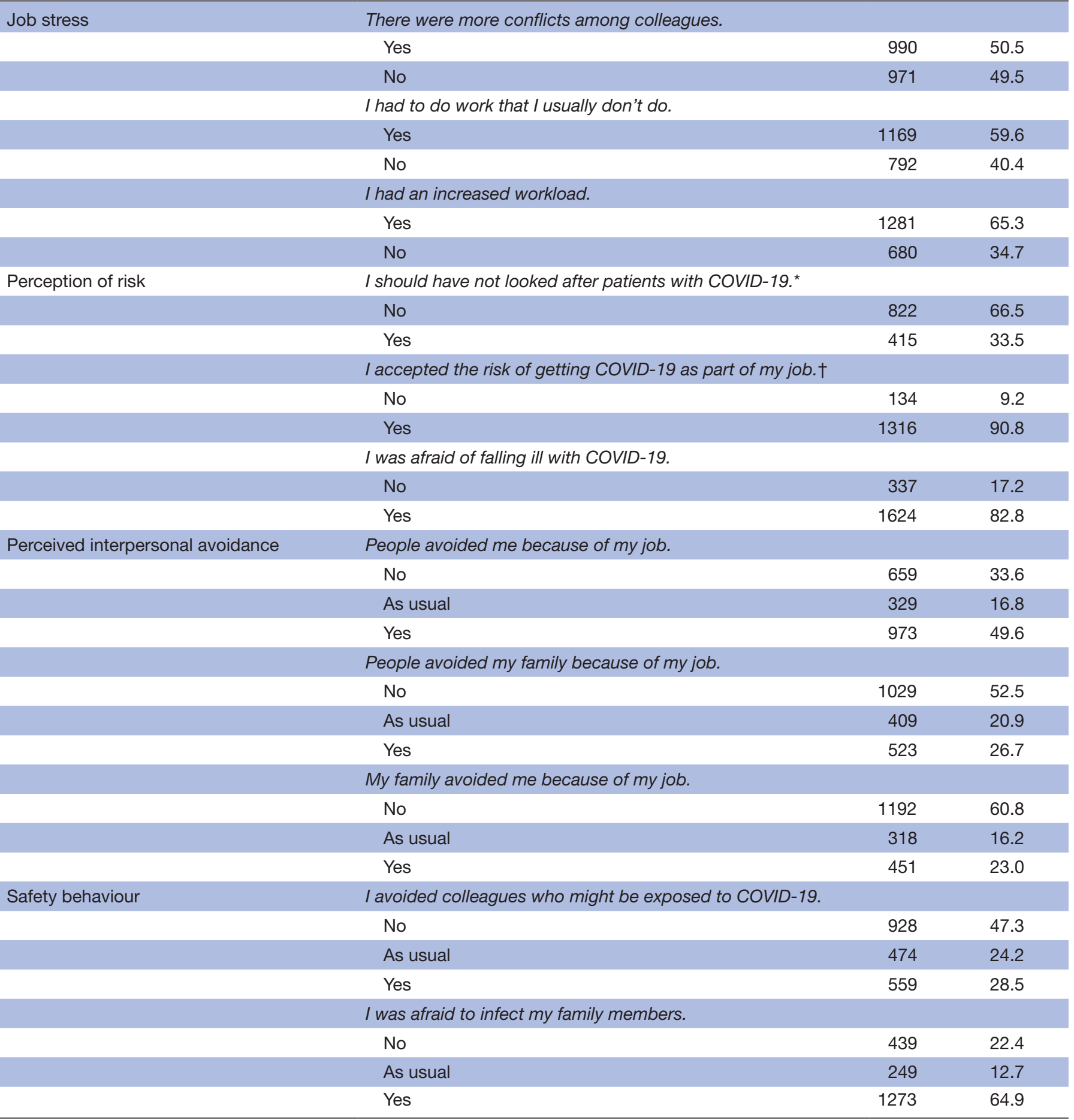

*724 subjects did not have contact with patients with COVID-19.

$\dagger 511$ subjects did not have contact with patients with COVID-19.

MBI-GS, Maslach Burnout Inventory-General Survey.

\section{Factors influencing healthcare workers' burn-out}

Univariate analyses estimating the association between each MBI-GS dimension and the various potential risk factors were first performed. Details are given in online supplemental part 3 .
Variables showing significant univariate associations were finally entered in multivariate analyses (see table 4). Several univariate associations lost their significance while other personal associations remained significant. 
Table 3 Personal and job-related characteristics across the three burn-out domains $(n=1961)$



${ }^{* \star *} \mathrm{P}<0.001 ;{ }^{* *} \mathrm{p}<0.01 ;{ }^{*} \mathrm{p}<0.05$.

†Infectious disease unit, pulmonary medicine, internal medicine units converted specifically to COVID-19.

‡Radiology and emergency department.

§Laboratory medicine, transfusion medicine, immunology, pathology, microbiology. 


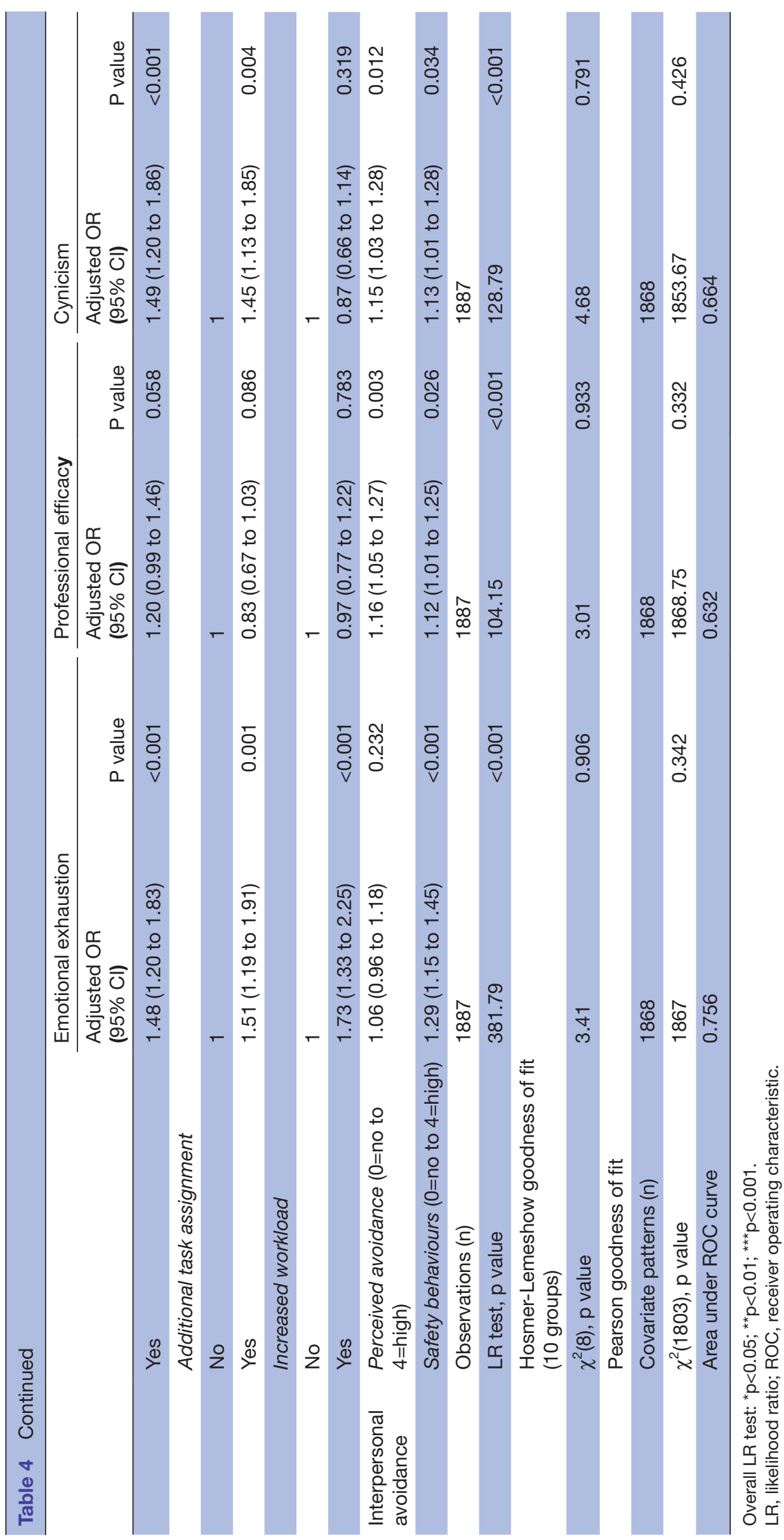

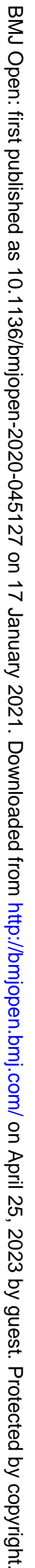


Work-related and COVID-related factors were entered together into the models.

Adjusted ORs indicated that being a woman (1.38; 95\% CI 1.06 to 1.79$)$, living alone $(1.70 ; 95 \%$ CI 1.27 to 2.28), having longer work experience (6-20years: 1.77 ; $95 \%$ CI 1.27 to 2.47 ; $>20$ years: 1.69 ; $95 \%$ CI 1.22 to 2.35 ), being a resident (1.82; 95\% CI 1.13 to 2.94$)$ and a nurse (1.75; 95\% CI 1.23 to 2.49 ) (in comparison to physician), having pre-existing psychological problems $(2.35 ; 95 \%$ CI 1.53 to 3.60), having experienced COVID-related traumatic event at work (2.40; 95\% CI 1.88 to 3.07$)$, having perceived more conflicts among colleagues at work (1.48; 95\% CI 1.20 to 1.83), having experienced more workload (1.73; 95\% CI 1.33 to 2.25), being assigned additional tasks $(1.51 ; 95 \%$ CI 1.19 to 1.91$)$ and having exhibited safety behaviours (1.29; 95\% CI 1.15 to 1.45$)$ increased the risk of high emotional exhaustion. On the other hand, working in front-line services dealing with patients with COVID-19 (0.61; 95\% CI 0.37 to 1.00$)$, in non-COVID wards $(0.53 ; 95 \%$ CI 0.37 to 0.77$)$ and in the administration $(0.53 ; 95 \%$ CI 0.29 to 0.96$)$ was associated with a reduced risk of emotional exhaustion as compared with working in ICUs.

The risk to experience low professional efficacy was grater for residents $(2.61 ; 95 \%$ CI 1.71 to 3.98$)$ in comparison to physicians, for those who had developed psychological problems before the pandemic outbreak (1.60; 95\% CI 1.08 to 2.36), those who had experienced social avoidance due to their profession (1.16; 95\% CI 1.05 to $1.27)$ and those who exhibited safety behaviours (1.12; $95 \%$ CI 1.01 to 1.25 ).

The risk of high cynicism was greater for residents (2.02; 95\% CI 1.24 to 3.27 ) and nurses $(1.69 ; 95 \%$ CI 1.17 to 2.45 ) as compared with physicians, for those who had pre-existing psychological problems $(1.84 ; 95 \%$ CI 1.23 to 2.75), for those who experienced COVID-related traumatic event at work $(1.37 ; 95 \%$ CI 1.06 to 1.76$)$, for those who perceived more conflicts among colleagues (1.49; 95\% CI 1.20 to 1.86), for those who were assigned additional tasks (1.45; 95\% CI 1.13 to 1.85$)$, those who had experienced social avoidance due to their profession $(1.15 ; 95 \%$ CI 1.03 to 1.28$)$ and those who exhibited safety behaviours $(1.13 ; 95 \%$ CI 1.01 to 1.28$)$. On the other hand, working in subintensive COVID-19 units $(0.47 ; 95 \%$ CI 0.28 to 0.77$)$ and non-COVID wards (0.60; $95 \%$ CI 0.42 to 0.87 ) was associated with a reduced risk of cynicism in comparison to working in ICUs.

\section{DISCUSSION}

Overall, we found that a relevant proportion of healthcare staff working in the Verona hospital reported symptoms of burn-out, and its impact was more burdensome for front-line healthcare workers: specifically, at least half of healthcare workers who provided either intensive or subintensive care to patients with COVID-19 reported high levels of emotional exhaustion and feelings of low professional efficacy, and $40 \%$ showed high levels of cynicism and detached attitude towards their job. This is a relevant finding. In fact, it should be noted that the percentage of front-line healthcare workers showing symptoms of burn-out in our study is considerably higher than those reported in Italy before the COVID-19 pandemic among staff working in a number of clinical settings, including ICUs, ${ }^{23-26}$ medical wards $^{27-30}$ and emergency units ${ }^{31}$ (see online supplemental part 4). It is thus reasonable to assume that the excess of workers experiencing high levels of burn-out found here, with respect to percentages reported in the literature, is due to the specific workrelated burden posed by the COVID-19 pandemic.

Our findings are consistent with research on past outbreaks of severe acute respiratory syndrome (SARS), Middle East respiratory syndrome and H1N1, reporting that front-line healthcare workers do experience considerable levels of burn-out. ${ }^{32}$ The finding that burn-out is more prevalent among front-line healthcare workers compared with staff working in non-COVID wards contrasts with a recent study conducted in China, reporting that frontline staff had a lower frequency of burn-out as compared with those working in usual wards. ${ }^{10}$ We may speculate that the healthcare system in China was more ready than other countries to tackle the challenge posed by the COVID-19 pandemic, having already developed protocols and procedures to counteract past outbreaks (eg, SARS or H1N1); therefore, Chinese healthcare workers might have had some expertise in managing such kinds of emergencies due to their involvement in previous infectious outbreaks.

It should be noted that it is not simply the direct contact with patients with COVID-19 per se a risk factor for developing burn-out, but rather the engagement with severely (or critically) ill patients with COVID-19 requiring subintensive or intensive care, since, for example, for emotional exhaustion, staff working in services dealing on daily basis with great numbers of patients with COVID-19 (such as radiology and emergency department) tend to display a lower risk of burn-out than staff working in subintensive or ICUs, and however the same risk of those working in non-COVID units. Therefore, to minimise the risk of burn-out during pandemic outbreaks, hospital administrations should recruit additional staff to unburden front-line healthcare workers from non-clinical tasks, and restrict excessive workload by scheduling breaks, limit work hours in subintensive and ICUs, and provide-when needed-regular psychosocial support.

We also found that nurses and resident physicians were the occupational categories more severely burdened by burn-out during the COVID-19 pandemic. Specifically, nurses displayed more emotional exhaustion, while residents were also at higher risk to experience a reduced sense of professional efficacy and to adopt detached attitudes towards their job. This is not an unexpected finding, since literature shows that nurses and residents are at increased risk of burn-out, in the light of the position they have within working organisation and the tasks they are generally assigned. This might have 
been amplified during the COVID-19 pandemic. In fact, previous studies have reported that nurses are particularly vulnerable to burn-out, and emergency nurses at an even higher risk, since emergency nursing is characterised by unpredictability, overcrowding and continuous confrontation with a broad range of diseases, injuries and traumatic events. ${ }^{33}$ During the COVID-19 pandemic, front-line nurses experienced sudden and dramatic challenges in the workplace in terms of increased workload, reassignment/redeployment to other roles or duties, infection threat, COVID-related traumatic events and frustration with the death of patients whom they care. This work overload may have contributed to burn-out among nurses by depleting their emotional capacity to meet the demands posed by the pandemic emergency. Thus, to minimise the risk of burn-out during pandemic outbreaks, hospital administrations should improve nurses' sense of control over their own scheduling and tasks by promoting their involvement in decision-making, particularly in demanding situations.

Previous research has also shown that the prevalence of burn-out is particularly high among residents. ${ }^{34}$ Intense work demands, limited control and a high degree of work-home interference strongly predispose resident physicians to burn-out. ${ }^{35}$ Resident physicians generally are the first-line health service providers, subjected to prolonged working hours, sleep deprivation and high job demands. Despite being young and inexperienced in the medical field, while also in the process of training, resident physicians normally treat patients with varying presentations and severity levels; at the same time, they are responsible for presenting reports to and taking orders from supervisors. Resident physicians are often confronted with the negative attitudes of their superiors, while also managing deceased and dying patients. High levels of responsibility coupled with role ambiguity and low levels of decisional autonomy are common among resident physicians and are associated with increased risk of burn-out. ${ }^{36}$ To minimise the risk of burn-out during pandemic outbreaks, hospital administrations should train and leverage the expertise of residents as front-line workers to handle critical patients and support and supervise them as long as needed.

Having a pre-existing psychological problem consistently increases the risk of burn-out. A recent study conducted in the general population found that people with pre-existing mental health conditions are more susceptible to stressors associated with COVID-19 and are more likely do adopt dysfunctional coping strategies (eg, self-isolation) compared with those with no mental health disorder. ${ }^{37}$ This process may also apply to healthcare workers, thus further increasing baseline levels of burn-out. Therefore, healthcare organisations should be aware that workers receiving treatment for ongoing psychological problems may be particularly vulnerable to the effect of adverse job conditions known to increase burn-out during pandemic emergencies. To minimise the risk of burn-out, hospital administrations should screen healthcare workers for ongoing psychological problems to protect the more vulnerable.

Having experienced a COVID-19-related traumatic event (eg, fear of becoming infected and/or infecting families, experiencing high mortality rates, grieving the loss of patients and colleagues, separation from families, changes in working practices and procedures, physical strain from prolonged wearing of personal protective equipment) increases the risk of burn-out, more specifically in terms of heightened emotional exhaustion and more detached attitude towards one's work. This is a relevant issue, since increased workplace stress resulting from COVID-related traumatic events, on the one hand, exacerbates baseline levels of burn-out and, on the other hand, when combined with underlying baseline burn-out may result in rising rates of post-traumatic stress disorders; thus, establishing and perpetuating a vicious cycle of chronic stress response. ${ }^{38}$ To minimise the risk of burn-out, hospital administrations should ensure the availability of appropriate and comfortable personal protective equipment to avoid infections among healthcare workers; ensure the safety and health of all staff members by the regular health surveillance including screening of infection; and empower staff by providing consistent and updated guidelines for managing patients through triage based on the case priority and severity. These actions are currently underway in our hospital.

A further risk factor for burn-out during the COVID-19 pandemic is interpersonal avoidance, which consistently impacted all the three MBI-GS dimensions in our study. This is a composite factor that addresses interpersonal avoidance or social rejection experienced by healthcare workers in their daily life due to their profession (eg, social stigma) and safety behaviours adopted by healthcare workers to protect themselves or their family members from potential sources of infection. Literature published during SARS and Ebola epidemics found that healthcare workers are often avoided, rejected and stigmatised due to their perceived link with the infection. ${ }^{39-41}$ Such experiences of social stigmatisation have detrimental effects on the personal life and emotional well-being of healthcare workers, as they may experience shame, guilt and sense of isolation. Also in the time of COVID-19, many healthcare workers have reported stigma from the public and family members. ${ }^{42}$ This, in turn, may have a negative effect on the relationship of healthcare workers with their job, in terms of reduced sense of efficacy and more detached attitude towards their job. Additionally, maladaptive coping strategies adopted both in the workplace and at home, such as avoidant safety behaviours, may contribute to increase employee burn-out and work disengagement. ${ }^{43}$ To minimise the risk of burn-out, hospital administrations should address work-life balance, promote connectedness and mutual support among healthcare workers and contrast social isolation and interpersonal avoidance. 


\section{Strengths and limitations}

The strengths of this study include the large sample size, the representativeness of the sample with respect to the overall hospital population, the wide array of occupational profiles considered and data collection performed during the lockdown period of the pandemic.

Among the possible limitations, we should consider the relatively low response rate. However, web-based surveys have generally lower response rates than faceto-face or telephone interviews or mail surveys, with a meta-analysis ${ }^{44}$ reporting a mean response rate similar to that of our study; it should also be noted that web-based surveys involving physicians or healthcare workers have even lower overall response rates. ${ }^{45}$ Another limitation is that we did not include personality traits among burn-out predictors addressed here. Moreover, other contextual and organisational variables not considered in the analyses might have exerted a significant role in the prediction of burn-out. In addition, the cross-sectional nature of the survey did not allow for the determination of causal relationships. Finally, the generalisability of our findings to other nations should be taken with caution, as the study was conducted in the specificity of the Italian national healthcare system.

\section{CONCLUSION}

Burn-out is a major concern for healthcare staff working in a large tertiary hospital during the COVID-19 pandemic and its impact is more burdensome for front-line junior physicians. This study serves to provide important evidence for the directing and promotion of mental well-being among healthcare workers and to prevent the sudden increase of burn-out in the event of a new COVID-19 healthcare emergency.

\section{Author affiliations}

${ }^{1}$ UOC Psichiatria, Azienda Ospedaliera Universitaria Integrata (AOUI) di Verona, Verona, Italy

${ }^{2}$ Section of Psychiatry, Department of Neuroscience, Biomedicine and Movement Sciences, University of Verona, Verona, Italy

${ }^{3}$ UOC Psicosomatica e Psicologia Medica, Azienda Ospedaliera Universitaria Integrata (AOUI) di Verona, Verona, Italy

${ }^{4}$ Section of Occupational Medicine, Department of Diagnostics and Public Health, University of Verona and Clinical Unit of Occupational Medicine, Integrated University Hospital of Verona, Verona, Italy

${ }^{5}$ Section of Hygiene, Department of Diagnostic and Public Health, University of Verona, Verona, Italy

${ }^{6}$ Health Director, Azienda Ospedaliera Universitaria Integrata (AOUI) di Verona, Verona, Italy

Acknowledgements The authors thank all the participants for their time and excellent cooperation in the survey. The authors also acknowledge Charley Nyzio for the linguistic revision of the final version of the manuscript.

Contributors AL, CBon and FA conceived and designed the study. CBon and AL designed the statistical analysis plan. AL, CBon and FA analysed the data and developed the figures and tables. AL, CBon, $F A, A C$ and $S P$ reviewed the findings and contributed to the interpretation. AL, CBon, ST, CBov and MR drafted the first version of the manuscript. All authors contributed to intellectual content during the drafting and revision of the work and approved the final version of the manuscript.

Funding The authors have not declared a specific grant for this research from any funding agency in the public, commercial or not-for-profit sectors.
Competing interests None declared.

\section{Patient consent for publication Not required.}

Ethics approval This study complies with ethical standards. The study was approved by the Ethics Committee of the Provinces of Verona and Rovigo (approval number 22002; 17 April 2020). All participants provided informed written consent.

Provenance and peer review Not commissioned; externally peer reviewed.

Data availability statement Data are available upon reasonable request.

Supplemental material This content has been supplied by the author(s). It has not been vetted by BMJ Publishing Group Limited (BMJ) and may not have been peer-reviewed. Any opinions or recommendations discussed are solely those of the author(s) and are not endorsed by BMJ. BMJ disclaims all liability and responsibility arising from any reliance placed on the content. Where the content includes any translated material, BMJ does not warrant the accuracy and reliability of the translations (including but not limited to local regulations, clinical guidelines, terminology, drug names and drug dosages), and is not responsible for any error and/or omissions arising from translation and adaptation or otherwise.

Open access This is an open access article distributed in accordance with the Creative Commons Attribution Non Commercial (CC BY-NC 4.0) license, which permits others to distribute, remix, adapt, build upon this work non-commercially, and license their derivative works on different terms, provided the original work is properly cited, appropriate credit is given, any changes made indicated, and the use is non-commercial. See: http://creativecommons.org/licenses/by-nc/4.0/.

ORCID iD

Antonio Lasalvia http://orcid.org/0000-0001-9963-6081

\section{REFERENCES}

1 Kisely S, Warren N, McMahon L, et al. Occurrence, prevention, and management of the psychological effects of emerging virus outbreaks on healthcare workers: rapid review and meta-analysis. BMJ 2020;369:m1642.

2 Pappa S, Ntella V, Giannakas T, et al. Prevalence of depression, anxiety, and insomnia among healthcare workers during the COVID-19 pandemic: a systematic review and meta-analysis. Brain Behav Immun 2020;88:901-7.

3 Chew NWS, Lee GKH, Tan BYQ, et al. A multinational, multicentre study on the psychological outcomes and associated physical symptoms amongst healthcare workers during COVID-19 outbreak. Brain Behav Immun 2020;88:559-65.

4 Bradley M, Chahar P. Burnout of healthcare providers during COVID-19. Cleve Clin J Med 2020. doi:10.3949/ccjm.87a.ccc051. [Epub ahead of print: 09 Jul 2020].

5 Leiter MP, Maslach C. Areas of worklife: a structured approach to organizational predictors of job burnout. In: Perrewé P, Ganster DC, eds. Research in occupational stress and well-being. Oxford, UK: Elsevier, 2003: 3. 91-134.

6 Maslach C, Jackson SE, Leiter MP. Maslach burnout inventory manual. 3rd ed. Palo Alto, CA: Consulting Psychologists Press, 1996.

7 Maslach C, Schaufeli WB, Leiter MP. Job burnout. Annu Rev Psychol 2001;52:397-422.

8 West CP, Dyrbye LN, Shanafelt TD. Physician burnout: contributors, consequences and solutions. J Intern Med 2018;283:516-29.

9 Hu D, Kong Y, Li W, et al. Frontline nurses' burnout, anxiety, depression, and fear statuses and their associated factors during the COVID-19 outbreak in Wuhan, China: a large-scale cross-sectional study. EClinicalMedicine 2020;24:100424.

10 Wu Y, Wang J, Luo C, et al. A comparison of burnout frequency among oncology physicians and nurses working on the frontline and usual wards during the COVID-19 epidemic in Wuhan, China. $J$ Pain Symptom Manage 2020;60:e60-5.

11 Barello S, Palamenghi L, Graffigna G. Burnout and somatic symptoms among frontline healthcare professionals at the peak of the Italian COVID-19 pandemic. Psychiatry Res 2020;290:113129.

12 Zerbini G, Ebigbo A, Reicherts P, et al. Psychosocial burden of healthcare professionals in times of COVID-19 - a survey conducted at the University Hospital Augsburg. Ger Med Sci 2020;18:Doc05.

13 Lasalvia A, Bonetto C, Porru S, et al. Psychological impact of COVID-19 pandemic on healthcare workers in a highly burdened area of north-east Italy. Epidemiol Psychiatr Sci 2020;30:e1.

14 Schaufeli WB, Leiter MP, Maslach C. Maslach Burnout InventoryGeneral Survey. In: Maslach C, Jackson SE, Leiter MP, eds. The Maslach burnout inventory. 3rd edn. Palo Alto, CA: Consulting Psychologists Press, 1996. 
15 Leiter MP, Schaufeli WB. Consistency of the burnout construct across occupations. Anxiety, Stress \& Coping 1996;9:229-43.

16 Bakker AB, Demerouti E, Schaufeli WB. Validation of the Maslach Burnout Inventory - General Survey: An Internet Study. Anxiety, Stress \& Coping 2002;15:245-60.

17 O'Connor K, Muller Neff D, Pitman S. Burnout in mental health professionals: a systematic review and meta-analysis of prevalence and determinants. Eur Psychiatry 2018;53:74-99.

18 Rotenstein LS, Torre M, Ramos MA, et al. Prevalence of burnout among physicians: a systematic review. JAMA 2018;320:1131-50.

19 Schaufeli WB, Van Dierendonck D. A cautionary note about the cross-national and clinical validity of cut-off points for the Maslach burnout inventory. Psychol Rep 1995;76:1083-90.

20 Lasalvia A, Bonetto C, Bertani M, et al. Influence of perceived organisational factors on job burnout: survey of community mental health staff. Br J Psychiatry 2009;195:537-44.

21 Maunder RG, Lancee WJ, Balderson KE, et al. Longterm psychological and occupational effects of providing Hospital healthcare during SARS outbreak. Emerg Infect Dis 2006;12:1924-32.

22 Imai T, Takahashi K, Hasegawa N, et al. Sars risk perceptions in healthcare workers, Japan. Emerg Infect Dis 2005;11:404-10.

23 Capuzzo M, Gilli G, Paparella L, et al. Factors predictive of patient satisfaction with anesthesia. Anesth Analg 2007;105:435-42.

24 Orena EF, Caldiroli D, Cortellazzi P. Does the Maslach burnout inventory correlate with cognitive performance in anesthesia practitioners? A pilot study. Saudi J Anaesth 2013;7:277-82.

25 Sanfilippo F, Noto A, Palumbo GJ, et al. Burnout in cardiac anesthesiologists: results from a national survey in Italy. $J$ Cardiothorac Vasc Anesth 2018;32:2459-66.

26 Vargas M, Spinelli G, Buonanno P, et al. Burnout among anesthesiologists and intensive care physicians: results from an Italian national survey. Inquiry 2020;57:46958020919263.

27 Grassi L, Magnani K. Psychiatric morbidity and burnout in the medical profession: an Italian study of general practitioners and hospital physicians. Psychother Psychosom 2000;69:329-34.

28 Quattrin R, Zanini A, Nascig E, et al. Level of burnout among nurses working in oncology in an Italian region. Oncol Nurs Forum 2006;33:815-20.

29 Klersy C, Callegari A, Martinelli V, et al. Burnout in health care providers of dialysis service in Northern Italy--a multicentre study. Nephrol Dial Transplant 2007;22:2283-90.

30 Bressi C, Manenti S, Porcellana M, et al. Haemato-oncology and burnout: an Italian survey. Br J Cancer 2008;98:1046-52.
31 Salvarani V, Rampoldi G, Ardenghi S, et al. Protecting emergency room nurses from burnout: the role of dispositional mindfulness, emotion regulation and empathy. J Nurs Manag 2019;27:765-74

32 Salazar de Pablo G, Vaquerizo-Serrano J, Catalan A, et al. Impact of coronavirus syndromes on physical and mental health of health care workers: systematic review and meta-analysis. J Affect Disord 2020;275:48-57.

33 Adriaenssens J, De Gucht V, Maes S. Determinants and prevalence of burnout in emergency nurses: a systematic review of 25 years of research. Int J Nurs Stud 2015;52:649-61.

34 Rodrigues $\mathrm{H}$, Cobucci R, Oliveira $\mathrm{A}$, et al. Burnout syndrome among medical residents: a systematic review and meta-analysis. PLoS One 2018;13:e0206840.

35 Thomas NK. Resident burnout. JAMA 2004;292:2880-9.

36 Prins JT, Gazendam-Donofrio SM, Tubben BJ, et al. Burnout in medical residents: a review. Med Educ 2007;41:788-800.

37 Asmundson GJG, Paluszek MM, Landry CA, et al. Do pre-existing anxiety-related and mood disorders differentially impact COVID-19 stress responses and coping? J Anxiety Disord 2020;74:102271.

38 Restauri N, Sheridan AD. Burnout and posttraumatic stress disorder in the coronavirus disease 2019 (COVID-19) pandemic: intersection, impact, and interventions. J Am Coll Radiol 2020;17:921-6.

39 Bai Y, Lin C-C, Lin C-Y, et al. Survey of stress reactions among health care workers involved with the SARS outbreak. Psychiatr Serv 2004;55:1055-7.

40 Maunder R, Hunter J, Vincent L, et al. The immediate psychological and occupational impact of the 2003 SARS outbreak in a teaching hospital. CMAJ 2003;168:1245-51.

41 Gee S, Skovdal M. Public discourses of Ebola contagion and courtesy stigma: the real risk to international health care workers returning home from the West Africa Ebola outbreak? Qual Health Res 2018;28:1499-508.

42 Bagcchi S. Stigma during the COVID-19 pandemic. Lancet Infect Dis 2020;20:782.

43 McCain RS, McKinley N, Dempster M, et al. A study of the relationship between resilience, burnout and coping strategies in doctors. Postgrad Med J 2018;94:43-7.

44 Cook C, Heath F, Thompson RL. A meta-analysis of response rates in Web- or Internet-based surveys. Educ Psychol Meas 2000;60:821-36.

45 Cunningham CT, Quan H, Hemmelgarn B, et al. Exploring physician specialist response rates to web-based surveys. BMC Med Res Methodol 2015;15:32 